\title{
EK Tra: a Spectroscopic Twin of VW Hyi
}

\author{
Boris T. Gänsicke, Klaus Beuermann \\ Universitäts-Sternwarte, Geismarlandstr. 11, Göttingen, Germany
}

Observations of the inter-outburst behaviour of dwarf novae can put constraints on theoretical models for the structure of the accretion disk and the outburst mechanism. The white-dwarf properties, such as temperature, postoutburst cooling timescale, and rotational velocity play a major rôle in the understanding of the processes in the boundary layer. Alas, the white dwarf could be detected directly only in a small number of dwarf novae. We have re-analysed the available $I U E$ observations of the southern dwarf nova EK TrA, testing the suggestion by Verbunt $(1987$, A\&AS 71,339$)$ that in this system the white dwarf is a significant source of UV radiation during quiescence.

We have decomposed the spectrum obtained closest to quiescence (Fig. 1) into accretion disc and white dwarf contribution following the method described by Gänsicke \& Beuermann (1996, A\&A 309, L47): we use the observed spectrum close to outburst maximum to describe the disc contribution and a grid $(\log g=$ 8.0 and $11000 \mathrm{~K} \leq T_{\mathrm{wd}} \leq 30000 \mathrm{~K}$, solar abundances) of calculated model spectra kindly provided by I. Hubeny for the white dwarf. Following our experience with VW Hyi (Gänsicke \& Beuermann, 1996, A\&A 309, L47), we allow for Gaussian emission lines of N V $\lambda 1240$, Si IV $\lambda 1400$ and C IV $\lambda 1549$. The observed spectrum was then fitted with the sum of these components using an evolutionary strategy algorithm. The best fit is achieved for a white dwarf of $T_{\mathrm{wd}}=16000 \mathrm{~K}$ and the accretion disc contributing $21 \%$ and $77 \%$ of the integrated SWP flux, respectively (Fig. 1). Assuming an $0.6 \mathrm{M}_{\odot}$ white dwarf, the scaling factor of the white dwarf model implies a distance of $\sim 140 \mathrm{pc}$, roughly twice that of VW Hyi. However, EK TrA was never observed in a truly quiescent state; at the time of the $I U E$ observations the residual accretion disc or accretion belt still dominates the UV light and the derived white-dwarf parameters remain necessarily uncertain. Comparison with VW Hyi teaches us that the decline of the spectrum towards Ly $\alpha$ should not, at face value, be taken as a direct measure of the white dwarf contribution to the overall flux.

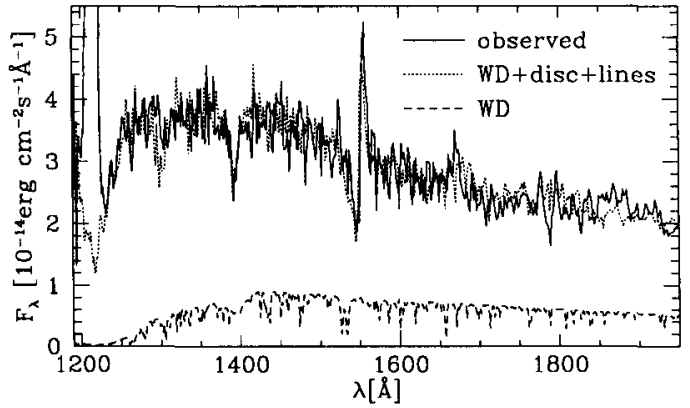

Fig.1. The best fit to the IUE (SWP09768) spectrum of EK TrA obtained shortly after the end of a superoutburst but clearly before the return into quiescence. 